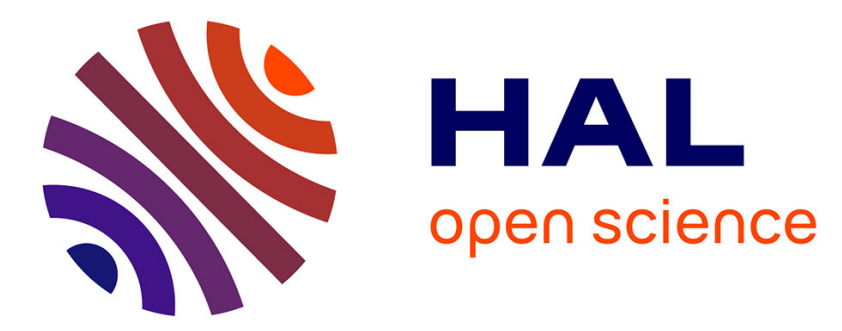

\title{
The Effect of Algorithm Form on Deformation and Instability in Tension
}

\author{
B. Goldthorpe, P. Church
}

\section{To cite this version:}

B. Goldthorpe, P. Church. The Effect of Algorithm Form on Deformation and Instability in Tension. Journal de Physique IV Proceedings, 1997, 07 (C3), pp.C3-753-C3-759. 10.1051/jp4:19973128 . jpa00255414

\section{HAL Id: jpa-00255414 https://hal.science/jpa-00255414}

Submitted on 1 Jan 1997

HAL is a multi-disciplinary open access archive for the deposit and dissemination of scientific research documents, whether they are published or not. The documents may come from teaching and research institutions in France or abroad, or from public or private research centers.
L'archive ouverte pluridisciplinaire HAL, est destinée au dépôt et à la diffusion de documents scientifiques de niveau recherche, publiés ou non, émanant des établissements d'enseignement et de recherche français ou étrangers, des laboratoires publics ou privés. 


\title{
The Effect of Algorithm Form on Deformation and Instability in Tension
}

\author{
B.D. Goldthorpe and P. Church \\ Defence Evaluation Research Agency, Fort Halstead, Sevenaoks, Kent TN14 7BP, U.K.
}

\begin{abstract}
Equilibrium and flow equations have been developed for limited boundary conditions to describe deformation and localisation in tension. These equations have been used to study the influence of algorithmic form on deformation response for four simple algorithms. It is shown that the structure of the algorithm can have a profound effect on the extent and rate of localisation. These results from analytical solutions are compared to those from computer modelling of similar problems and the agreement is shown to be extremely good over the whole range.
\end{abstract}

\section{INTRODUCTION}

Constitutive equations are now in widespread use to describe the deformation behaviour of solids both in theoretical work and in computer modelling. These algorithms range from forms having a sound physical basis and accurately determined coefficients such as the MTS [1] and modified Armstrong-Zerilli [2,3] models to those where the form and range are less well founded and have poorer predictive capability.

The main aim of this paper is to study the role of algorithm form in controlling deformation behaviour in particular the tendency to promote or resist localised deformation. An improved understanding of algorithmic form should provide a valuable guide in the construction of new algorithms and an essential guide to the selection and development of new materials which give a desired response to deformation.

The approach used here is primarily analytical and applied to tension problems initially to develop equilibrium conditions in flow which provide instability criteria in terms of the boundary conditions and the algorithm. These methods can also be used to develop equations to describe flow, that is shape as a function of time, but for reasons of space this development has not been included. For the above reasons the description of flow has been limited to the early stages of deformation with simplified boundary conditions.

Some of the effects observed were surprisingly large and have not previously been reported. It was partly to verify these results that computer modelling studies were carried out using the same algorithms. The agreement between theory and computer modelling was excellent both qualitatively and quantitatively where a full quantitative comparison could be made.

\section{EQUILIBRIUM CONDITIONS FOR FLOW IN TENSION}

In general the forrce on a rod in tension can be described by

$$
P=\pi a^{2} \lambda \bar{\sigma}
$$

where $a$ is the radius, $\bar{\sigma}$ is the constitutive equation and $\lambda$ is the constraint factor defined by

$$
\lambda=\frac{2 \int_{0}^{a} r \sigma_{z} d r}{a^{2} \bar{\sigma}}
$$

where $\sigma_{z}$ is the axial stress.

In considering the deformation of this rod we shall confine our attention to a small region at the centre of an instability centred at $z=0$ or incipient instability defined by its radius of curvature $\rho$ where

$$
\frac{1}{\rho}=\frac{d^{2} a}{d z^{2}}
$$

For the early stages of deformation $\lambda$ for a necked rod can be calculated using Bridgman's equation [4]. Deformation of this region will involve a change in axial position for any Lagrangian point. Thus a cylinder of initial dimensions $\left(a_{0}, 2 z_{0}\right)$ and current dimension $(a, 2 z)$ will by constant volume give

$$
a^{2} z=a_{0}^{2} z_{0}
$$




$$
\frac{d z}{d z_{0}}=\frac{a_{0}^{2}}{a^{2}} \quad \text { as } z \rightarrow 0
$$

Differentiation w.r.t. $z$ during deformation will require the application of this transformation.

\subsection{Boundary Conditions}

If for simplicity we use one of the most basic algorithms

and substitute in equation (1) we have

$$
\bar{\sigma}=C \dot{\varepsilon}^{m}
$$

and since

$$
P=\pi a^{2} \lambda C \dot{\varepsilon}^{m}
$$

$$
\dot{\varepsilon}=-\frac{2}{a} \frac{d a}{d t}
$$

equation (1) can be integrated to give

$$
-\frac{1}{2} \int_{t_{0}}^{t}\left(\frac{P}{\pi}\right)^{Q} d t=\int_{a_{0}}^{a} a^{2 Q-1} \lambda^{Q} d a
$$

where $Q=1 / m$.

Since the 1.h.s. is constant with $z$ differentiation twice w.r.t. $z$ gives the general form

$$
\frac{a^{2 Q-6} \lambda^{Q}}{\rho}-\frac{a_{0}^{2 Q-6} \lambda_{0}}{\rho_{0}}+Q \int a^{2 Q-6} \lambda^{Q-1} \frac{\partial^{2} \lambda}{\partial z^{2}} d a=0
$$

This equation defines some important boundary conditions for the problem. If the rod is a tensile specimen with a parallel gauge length then $a_{0}$ is not a function of $z$ and the second term disappears. Thus the development of instability defined in the first term depends on the variation of $\lambda$ with $z$. In this case it will be the constraint induced by the enlarged ends which causes a variation in $\bar{\sigma}$ along the length.

If, however, the test is idealised so that the initial constraint is zero then localisation can only occur if $a_{0}$ varies with $z$ and the development of instability is simply described by the first two terms in equation (8).

Because of the uncertainty and difficulty of representing initial constraint any further analysis will use an assumed $\rho_{o}$ without affecting the generality of the results.

\subsection{Localisation Criteria}

The principal condition for localisation is given by the equilibrium along the axis. For this we differentiate equation (1) twice w.r.t. $z$ at constant time to give

$$
\frac{1}{\lambda} \frac{d^{2} \lambda}{d z^{2}}+\frac{2}{a \rho}+\frac{1}{\bar{\sigma}} \frac{\partial^{2} \bar{\sigma}}{\partial z^{2}}=0
$$

It is immediately apparent that if

$$
\frac{1}{\bar{\sigma}} \frac{\partial^{2} \bar{\sigma}}{\partial z^{2}}=-\frac{2}{a \rho}
$$

then no change in constraint is necessary and deformation is perfectly stable. Equation (9), therefore, defines the conditions for localisation since any movement from equilibrium will change the value of $\rho$. If (9) is positive then $\rho$ will decrease and localisation will increase. This equation is quite general in terms of constrains since external constraints as well as those arising from localisation will contribute. At the start of a tensile test $\rho$ is infinite and so it defines the condition when necking will start from end constraints for a particular material. Equation (10) is adequate for initial curvature boundary conditions since all that is required is that the constraint due to $\rho$ remains constant. Before we can proceed any further with the analysis $\bar{\sigma}$ must be replaced by a detailed algorithm. This will require two more results which will now be developed. The first is an alternative form for $\partial^{2} \varepsilon / \partial z^{2}$

$$
\text { Since } \varepsilon=\ln \left(\frac{a_{0}^{2}}{a^{2}}\right)
$$


Then

$$
\frac{\partial^{2} \varepsilon}{\partial z^{2}}=-\frac{2}{a \rho}
$$

The second and more difficult problem is an alternative form for $\frac{1}{\dot{\varepsilon}} \frac{\partial^{2} \dot{\varepsilon}}{\partial z^{2}}$. For this we use the identity

and differentiate twice w.r.t. $z$. This gives

$$
\int_{t_{0}}^{t} \dot{\varepsilon} d t=\int_{\varepsilon_{0}}^{\varepsilon} d \varepsilon
$$

$$
\int_{t_{0}}^{t} \frac{a_{0}^{4}}{a^{4}} \frac{\partial^{2} \dot{\varepsilon}}{\partial z^{2}} d t=\frac{\partial^{2} \varepsilon}{\partial z^{2}} \frac{a_{0}^{4}}{a^{4}}-\frac{\partial^{2} \varepsilon_{0}}{\partial z_{0}^{2}}=-\frac{2}{a \rho} \frac{a_{0}^{4}}{a^{4}}-\frac{2}{a_{0} \rho_{0}}
$$

Differentiating w.r.t $t$ and cancelling $a$ and $a_{0}$ gives

$$
\frac{\partial^{2} \dot{\varepsilon}}{\partial z^{2}}=-\frac{2}{a} \frac{d \frac{1}{\rho}}{d t}+\frac{10}{a^{2} \rho} \frac{d a}{d t}=-\frac{2}{a} \frac{d \frac{1}{\rho}}{d a} \frac{d a}{d t}+\frac{10}{a^{2} \rho} \frac{d a}{d t}
$$

and from equation (6) we have

$$
\frac{1}{\dot{\varepsilon}} \frac{\partial^{2} \dot{\varepsilon}}{\partial z^{2}}=\frac{d \frac{1}{\rho}}{d a}-\frac{5}{a \rho}=\frac{1}{a} \frac{d \frac{a}{\rho}}{d a}-\frac{6}{a \rho}
$$

\section{EFFECT OF ALGORITHMIC FORM}

\subsection{Instability Conditions}

In this section the instability conditions for four different algorithms will be developed using equations (9), (11) and (13).

\subsubsection{Visco-Plastic Solid}

The algorithm for this is given by equation (5). Differentiating twice w.r.t. $z$ and dividing by $\bar{\sigma}$ and $m$ leads to equation (13). Substitution into equation (9) gives

$$
\frac{1}{\lambda} \frac{\partial^{2} \lambda}{\partial z^{2}}+\frac{2}{a \rho}+m\left(\frac{1}{a} \frac{d \frac{a}{\rho}}{d a}-\frac{6}{a \rho}\right)=0
$$

Now if the boundary conditions are such that only $a_{0}$ is a function of $z$ then the first term is negative and $\frac{d \frac{a}{\rho}}{d a}$ is negative for neck growth. Thus if $m \geq 0.33$ then there will be no neck formation or if a neck aiready exists further deformation will remove it. If $m<0.33$ then neck formation will occur and the rate of growth is given by

$$
\frac{1}{a} \frac{d \frac{a}{\rho}}{d a}=-\frac{1}{m}\left[\frac{1}{\lambda} \frac{\partial^{2} \lambda}{\partial z^{2}}+\frac{2-6 m}{a \rho}\right]
$$

Clearly the neck growth rate is roughly inversely proportional to $m$ but an accurate solution of this and subsequent equations is, for reasons of space, beyond the scope of this paper.

If the boundary conditions are such that there is end constraint as in a tensile test then $\frac{1}{\lambda} \frac{\partial^{2} \lambda}{\partial z^{2}}$ will normally be initially positive. This will require $m$ to be somewhat greater than 0.33 for perfect stability and will lead to slightly higher neck growth rates.

\subsubsection{Visco-Plastic Solid With Additive Constant}

This material is represented by the algorithm

$$
\bar{\sigma}=C_{1}+C_{2} \dot{\varepsilon}^{m}
$$

Again differentiating twice w.r.t. $z$ and dividing by $\bar{\sigma}$ gives 


$$
\frac{1}{\bar{\sigma}} \frac{\partial^{2} \bar{\sigma}}{\partial z^{2}}=\frac{m}{\dot{\varepsilon}} \frac{\bar{\sigma}-C_{1}}{\bar{\sigma}} \frac{\partial^{2} \dot{\varepsilon}}{\partial z^{2}}=m\left(1-\frac{C_{1}}{\bar{\sigma}}\right)\left(\frac{1}{a} \frac{d \frac{a}{\rho}}{d a}-\frac{6}{a \rho}\right)=\frac{m C_{2} \dot{\varepsilon}^{m}}{\bar{\sigma}}\left(\frac{1}{a} \frac{d \frac{a}{\rho}}{d a}-\frac{6}{a \rho}\right)
$$

Using the boundary conditions with only $a_{0}$ as a function of $z$ we can consider the behaviour of equation (9) after substitution of equation (16) i.e.

$$
\frac{1}{\lambda} \frac{\partial^{2} \lambda}{\partial z^{2}}+\frac{2}{a \rho}+m \frac{C_{2} \dot{\varepsilon}^{m}}{\bar{\sigma}}\left(\frac{1}{a} \frac{d \frac{a}{\rho}}{d a}-\frac{6}{a \rho}\right)=0
$$

This equation shows quite different characteristics to (13) since it is only when $C_{1}$ is small compared to the strain rate term that high levels of stability can be approached for any realistic value of $m$ i e. $m \leq 1$. In general, therefore, necking occurs earlier in this model and proceeds more rapidly than the visco-plastic model. Furthermore there is a significant strain rate effect since for given values of $C_{1}$ and $C_{2}$ the last term in equation (17) increases in absolute value and therefore increases stability and decreases neck growth rates. In the limit as $m \rightarrow \infty$ this model becomes a viscous solid and as $C_{2} / C_{1} \rightarrow 0$ it becomes a rigid plastic solid irrespective of the value of $m$.

\subsubsection{Visco-Plastic Solid With Coupled Strain Hardening}

This material is represented by the algorithm

$$
\bar{\sigma}=C \varepsilon^{n} \dot{\varepsilon}^{m}
$$

and is often used as an approximate model for real solids. Using similar methods to section 3.1 we use equations (11) and (13) and (9) to obtain

$$
\frac{1}{\lambda} \frac{\partial^{2} \lambda}{\partial z^{2}}+\frac{2}{a \rho}-\frac{2 n}{\varepsilon a \rho}+m\left(\frac{1}{a} \frac{d \frac{a}{\rho}}{d a}-\frac{6}{a \rho}\right)=0
$$

The overall condition for perfect stability is that $n / \varepsilon+3 m \geq 1$.

Clearly if $n=0$ then the model and deformation response is identical to equation (14). If $m$ is zero then deformation is perfectly stable for $\varepsilon \leq \mathrm{n}$ in agreement with the Considère criterion [5]. If, however, both $m$ and $n$ are greater than zero then this model is more stable than equation (14) for all values of strain. This is important if this model is used to represent real materials since unrealistically high levels of stability and low neck growth rates are shown unless $m$ is very low.

\subsubsection{Visco-Plastic Solid With Uncoupled Strain Hardening}

The algorithm for this material is given by

$$
\bar{\sigma}=C_{1} \varepsilon^{n}+C_{2} \dot{\varepsilon}^{m}
$$

Again using equation (11) and (13) and (9) similar methods to section 3.1 give

$$
\frac{1}{\lambda} \frac{\partial^{2} \lambda}{\partial z^{2}}+\frac{2}{a \rho}-\frac{2 n}{\varepsilon a \rho} \frac{C_{1} \varepsilon^{n}}{\bar{\sigma}}+\frac{m C_{2} \dot{\varepsilon}^{m}}{\bar{\sigma}}\left(\frac{1}{a} \frac{d \frac{a}{\rho}}{d a}-\frac{6}{a \rho}\right)=0
$$

Again if $m$ is zero then perfect stability is shown when $\varepsilon \leq \mathrm{n}$. If $m$ is greater than zero deformation behaviour is similar to the model in equation (15) since it is the relative values of $C_{1}$ and $C_{2}$ that determine stability for realistic values of $m$ and $n$. In particular as the value of $C_{1}$ increases necking starts earlier and localisation rates are higher. This is particularly important in this model since it is a good representation of steel and increases in the strength of steel are achieved through increases in both the $C_{1}$ value in equation (15) and in equation (20). Thus increasing the strength of materials represented by these models leads to a rapid increase in localigation rates.

\subsection{Rates Of Localisation}

As has already been stated the complete solution of the equilibrium and flow equations will not be described here. The early stages of deformation can, however, be described for constant load conditions by means of equation (8). Under the boundary conditions without end constraint equation (8) reduces to

$$
a^{2 Q-6} \lambda^{Q} \frac{a}{\rho}=a_{0}^{2 Q-6} \lambda_{0}^{Q} \frac{a_{0}}{\rho_{0}}
$$

and this is the flow equation for the viscous solid.

By exactly the same methods the algorithm in equation (18) gives 


$$
a^{2 Q-6} \varepsilon^{Q_{n}} \lambda^{Q} \frac{a}{\rho}=a_{0}^{2 Q-6} \varepsilon_{0}^{Q n} \lambda_{0}^{Q} \frac{a_{0}}{\rho_{0}}
$$

Again using the methods of section 2.1 the algorithm in equation (15) can be written

and the flow equation is then

$$
-\frac{d t}{2}=\frac{a^{2 Q-1} \lambda^{Q}}{\left(P-\pi a^{2} C_{1}\right)^{Q}} d a
$$

$$
\frac{a^{2 Q-6} \lambda^{Q}}{\left(P-\pi a^{2} C_{1}\right)^{Q}} \frac{a}{\rho}=\frac{a_{0}^{2 Q-6} \lambda_{0}^{Q}}{\left(P-\pi a_{0}^{2} C_{1}\right)^{Q}} \frac{a_{0}}{\rho_{0}}
$$

Equations (22) and (23) have been used to calculate early flow curves and some results are shown in figure 1 where $a^{\prime} \rho$ is plotted against strain. For viscous solids with $m=0.1$ and $m=0.2$ it can be seen that for an $a_{0} / \rho_{0}$ of 0.004 localisation starts immediately. Initially neck growth is slow but steadily accelerates to an eventually steep curve and the shape of the whole curve is strongly dependent on $m$. For $m=0.2$ only moderate necking occurs up to strains of 2 . If $m=0.33$ no localisation occurs as shown by both equations (14) and (22). Figure 1 also shows a curve for a viscous solid with coupled strain hardening. This shows that no localisation occurs up to a strain of 0.5 as would be expected from a simple strain hardening solid. In the case of this combined model, however, there is negligible neck growth up to a strain of 0.7 but at strains greater than this neck growth accelerates rapidly. This is characteristic of copper for which this model is sometimes used.

Figure 2 shows curves calculated using equation (23) for a viscous solid with additive constant. These curves show the remarkable effect of the constant in accelerating localisation and the role of strain rate in this process. It is evident from figure 2 that the effect of the constant decreases as the strain rate increases and the effect disappears as the strain rate approaches infinity. These observations are in agreement with the more rigorously derived equation (17) and the observations also apply to the strain hardening solid in equation (21).

\section{COMPUTER MODELLING}

Although, of necessity, very little space can be devoted to computer modelling in this paper it should be emphasised that it has played a major role throughout this work. The combination of analytical and computer modelling studies of many problems is particularly powerful since analysis can develop relationships and identify mechanisms. These can then be used to provide boundary conditions for the computer modelling which can be used both to verify the results of analysis and extend the work to more intractable problems.

This approach was used here using computational techniques based on the Lagrangian hydrocode DYNA 2D and the actual methods have been reported in detail elsewhere [6]. The first modelling studies were of viscous solids in tension and plots of various models after deformation are shown in figure 3 . The model with $\mathrm{m}=0.55$ shows perfect stability in agreement with the analytical developments in the paper. The model with $\mathrm{m}=0.4$ shows some slight localisation but very large extension without further localisation. We also take this as support for the analytical work since the large end constraint in this short gauge length specimen would initially provide a positive imbalance in equation (14) which allows localisation to develop. As deformation proceeds the constraint is reduced and conditions move towards indefinite stability.

Figure 3 also shows models for $\mathrm{m}=0.1$ and $\mathrm{m}=0.2$ which exhibit localisation increasing with decreasing $\mathrm{m}$. Some modelling points are also shown in figure 1 for $\mathrm{m}=0.2$ which give good quantitative agreement with the calculated early growth curves. A modelling point is also shown in figure 1 for the coupled strain hardening solid and this also shows good agreement.

Modelling studies were also carried out on viscous solids with additive constants. One of the more dramatic results is shown in figure 4 for a model with $m=0.55$ and $C_{1} / C_{2}=800$. Without the constant the model is extremely stable as shown in figure 3 but the effect of the constant is to produce very early localisation even at the high strain rate of $10^{3} \mathrm{sec}^{-1}$ used here.

\section{DISCUSSION}

One of the most significant results of the work described above is the importance of the form of the algorithm (as opposed to the specific value of the coefficients) on deformation behaviour. The algorithmic forms used can be regarded as some of the basic building blocks of constitutive equations that are currently used eg [1], [3]. It appears that whatever expressions are used to describe strain hardening and strain rate 
sensitivity along with various constants a factor of at least equal importance is how these components are related to each other. This is of particular significance in the area of strain localisation since the same basic relationships in different forms can give a good description of deformation over a narrow range of variables or under particular boundary conditions. Where the boundary conditions lead to localisation. however, the deformation response can show wide divergence depending on the form.

Although the work described here is confined to the effect of strain rate, strain and constraint in tension parallel work by the authors has shown that the effects of temperature and other modes of deformation can be treated in the same way. These investigations have led to similar conclusions on the care needed in the choice of algorithms to describe deformation. It would appear that if these algorithms are not physically based or founded on empirical verification over a wide range then studies of complex processes such as adiabatic shear and ductile fracture may leave some of their conclusions open to doubt.

\section{REFERENCES}

[1] Follansbee PS and Kocks UF, Acta Met 36, 1988, pp81-93

[2] Armstrong RW and Zerilli FJ, Jnl de Phys 49, 1988, pC3-529

[3] Goldthorpe BD, Jnl de Phys 1, 1991, pC3-829

[4] Bridgman PW, Large Plastic Flow and Fracture, McGraw-Hill, 1952

[5] Considère A, Ann Ponts Chaussée 9, 1885, p574

[6] Church P, Dyna 3D User Group Conference, 1992

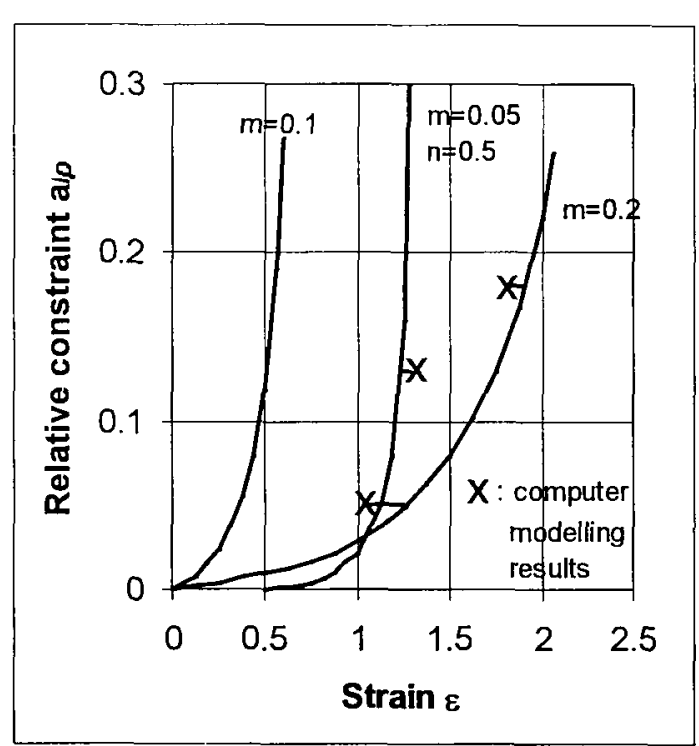

Figure 1; early neck growth curves for viscous solid; computer modelling results

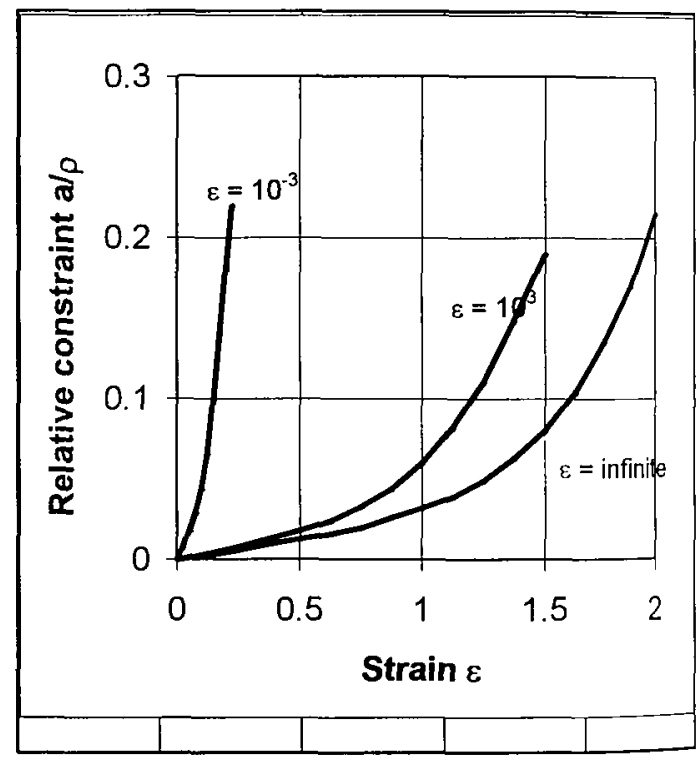

Figure 2; Effect of strain rate for viscous solid with additive constant; $m=0.2, C_{1} / C_{2}=1$ 

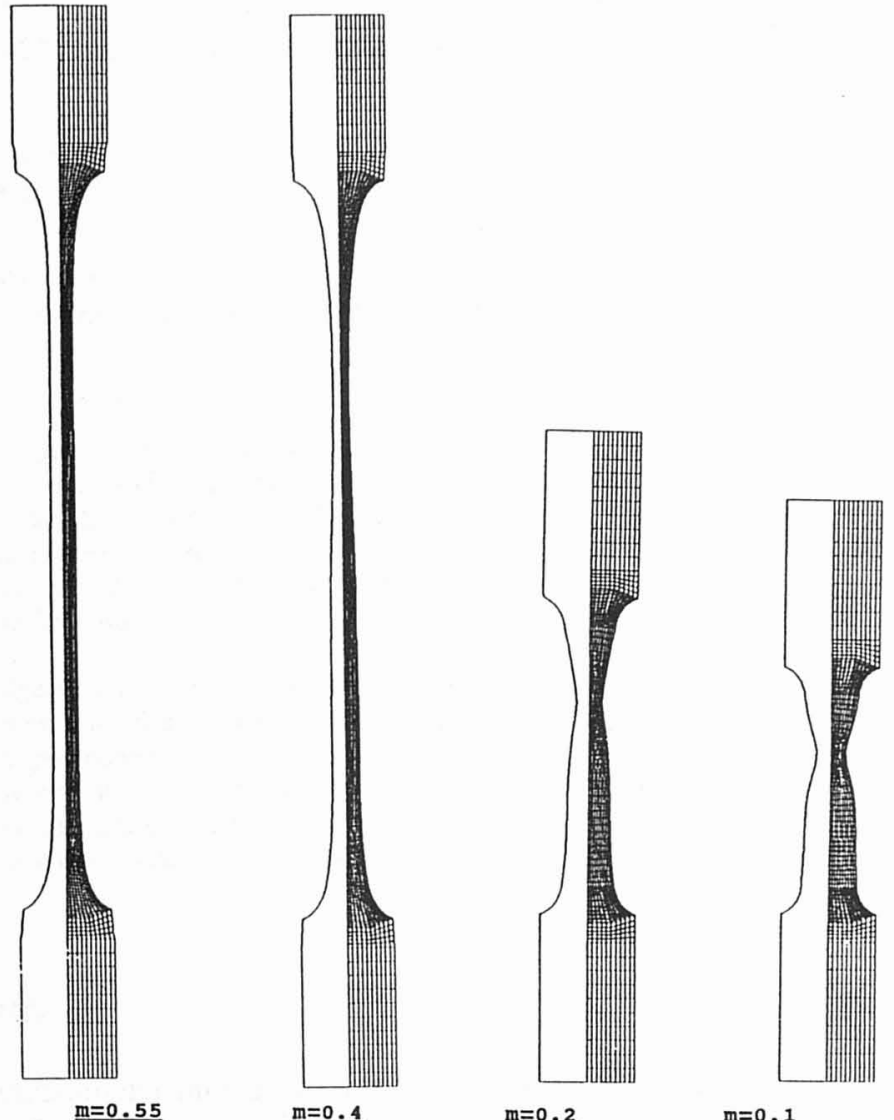

lïgure 3; Modelling Results of Localisation for Different $m$ Values

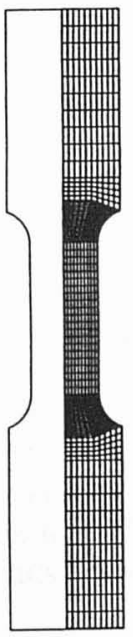

(ii) undeformed

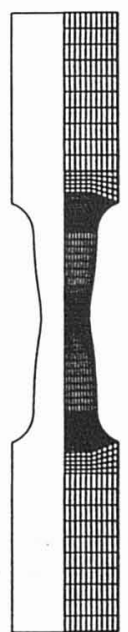

(h) carh lecalisation

lïgure 4; Effect of Rate Independent Constant for $m=0.55, C_{1} / C_{2}=800$ 